\title{
Heterogeneous Microtubules of Self-assembled Silver and Gold Nanoparticles Using Alive Biotemplates
}

\author{
Andressa Mayumi Kubo ${ }^{\circledR}$, Luiz Fernando Gorup ${ }^{a}$, Luciana Silva Amaral ${ }^{a}$,Edson Rodrigues-Filho ${ }^{a}$, \\ Emerson Rodrigues de Camargo ${ }^{*} *$ (우 \\ ${ }^{a}$ Universidade Federal de São Carlos - UFSCar, Rod. Washington Luis km 235, CP 676, 13565-905, \\ São Carlos, SP, Brazil
}

Received: October 19, 2017; Revised: February 14, 2018; Accepted: April 04, 2018

\begin{abstract}
Microtubules were constructed by covering the fungus Aspergillus aculeatus sequentially with silver and gold nanoparticles, resulting in a stable hybrid mesostructured material that presented three distinct regions containing different combinations of silver and gold nanoparticles. These heterogeneities were determined by the hyphal growth since the impossibility to cover the dead fungus, which suggests the influence of the secondary metabolites produced by living fungus in the deposition mechanism.
\end{abstract}

Keywords: biotemplates, self assembly, nanoparticles.

\section{Introduction}

Biotechnological and supramolecular systems can range from a few nanometers to about one micrometer, which makes possible to manufacture heterostructures of large morphological and functional complexity. Among these systems, micrometric and nanometric materials constructed using biotemplates made of bacteria, ${ }^{1,2} \mathrm{DNA}^{3,4}$ or fungi ${ }^{5,6}$ have attracted a great interest due their unusual morphologies. Because of their reproducibility and variety of structures associated with a precise morphological control, biotemplates became an efficient alternative to overcome the limitations of conventional materials.

Self-assembled three-dimensional hybrid materials can be fabricated using non-functionalized inorganic nanoparticles as building blocks, such as noble metal nanoparticles obtained with simple and well-established techniques. ${ }^{7-10}$ These nanoparticles are widely used to obtain materials where shape homogeneity and composition are quite important, for example in sensors, catalysis and devices for medical and engineering applications. ${ }^{11-15}$

Heterostructures consisting of multilayers with controlled thickness have been constructed owing to interactions between nanoparticles and biotemplates. ${ }^{16,17}$ Particularly, fungi can be effectively used as biotemplates since they are skilled at adapting in distinct environments. This versatility extends to their peculiar shapes, ranging from mushrooms to microfilaments. ${ }^{18,19}$ However, studies in relation to their uncontrolled growing after the contact with noble metal nanoparticles and the possibility to form microtubules containing different types of nanoparticles are still limited. ${ }^{20}$ Therefore, understanding how nanoparticles form multilayers on the fungi surface opens up the opportunity to study comprehensively the physical and chemical characteristics of bioinorganic interfaces. In this context, we are reporting the construction of hybrid materials using biotemplates made of fungus Aspergillus aculeatus that were covered with nonfunctionalized nanoparticles of silver and gold and we are proposing a model to explain the origin of the compositional heterogeneities observed.

\section{Experimental}

Silver and gold nanoparticles were synthesized by the Turkevich method ${ }^{7}$. Precursors were gold (III) chloride trihydrate (99.9 \% trace metal basis, Sigma-Aldrich) and silver nitrate ( $\geq 99.0 \%$ ACS reagent, Sigma-Aldrich). Sodium citrate was used as stabilizer and reducing agent. All of the chemicals were used as received. Water was previously deionized using a Millipore Elix-3 equipment. All the solutions and deionized water were autoclaved at $120^{\circ} \mathrm{C}$ for 30 minutes. The synthesis of all samples, including gold and silver colloidal nanoparticles, were prepared under UV-Vis light inside a laminar flow chamber that was strictly sterilized with sodium hypochlorite and ethylic alcohol 70 $\%$. Solutions of silver and gold salts, with the concentration of $0.1 \mathrm{~mol} \cdot \mathrm{L}^{-1}$ and volume of $1 \mathrm{~mL}$, were prepared with deionized water. These solutions were heated until boiling temperature under constant agitation. After approximately one minute, $1 \mathrm{~mL}$ of sodium citrate with the concentration of $0.3 \mathrm{~mol} \cdot \mathrm{L}^{-1}$ was added into silver and gold solutions to form nanoparticles. The flask containing silver was cooled to room temperature after 5 minutes of reaction and the other 
with gold was cooled after 12 minutes. The XRD patterns of silver and gold nanoparticles and their respective UV-Vis absorption spectra are shown in the Supplementary Material (F1gure SI).

Fungal hyphae were grown in $25 \mathrm{~mL}$ of an autoclaved culture medium specific for fungi by adding $200 \mu \mathrm{L}$ of the suspension with activated spores. After hyphae growth, the culture medium was removed, which was followed by the addition of the noble metal colloidal dispersions. Microtubules were prepared containing sequentially silver (AgNP) and gold (AuNP) nanoparticles. Microtubules constructed with multilayers of metal nanoparticles were prepared aiming to obtain stable hybrid materials constituted of walls made of silver and gold nanoparticles. In this experiment, $100 \mathrm{~mL}$ of the silver colloidal dispersion, previously synthesized, were added into the mycelial mass. After 7 days, this colloidal dispersion of silver nanoparticles was removed using a peristaltic pump and the mycelial material was washed exhaustively with deionized water. Then, $100 \mathrm{~mL}$ of a second colloidal dispersion of gold nanoparticles were added. This second colloidal dispersion was kept in contact to the mycelial mass for another 7 days. Finally, this colloidal dispersion of gold nanoparticles was removed, leaving the hybrid material made of fungus covered with two different nanoparticles. The hybrid material was characterized by field emission gun-scanning electron microscopy (FEG-SEM) and energy dispersive X-ray spectroscopy (EDX).

\section{Results and Discussion}

The germination of spores and the hyphae growth of the fungus Aspergillus aculeatus in a culture medium rich in nutrients resulted in a microtubular structure suitable to be used as a biotemplate. Figure 1a shows an optical micrograph of the filamentous hyphae before the addition of nanoparticles in which is observed the hyphae width homogeneity. After the period necessary for hyphal growth, culture medium was removed and the mycelial mass, which was exhaustively washed with deionized water, was kept inside the Erlenmeyer flask. Then, the first colloidal dispersion, containing only AgNP, was added into this flask, which means that nutrients were no longer available in the medium to allow the hyphae growth, except by the citrate used in excess to reduce the silver ion and to stabilize the silver nanoparticles. Self-organization of AgNP took place on the surface of the hyphae, resulting in an opaque grayish yellow filamentous structure that could be easily separated from the aqueous medium (Fig.1b).

The interaction between the fungus and silver nanoparticles was strong enough to form structures completely covered by nanoparticles that were easily handled because of their mechanical strength. ${ }^{21}$ The first layer of silver nanoparticles was probably formed through covalent bonds between the metal nanoparticles and the proteins of the fungus
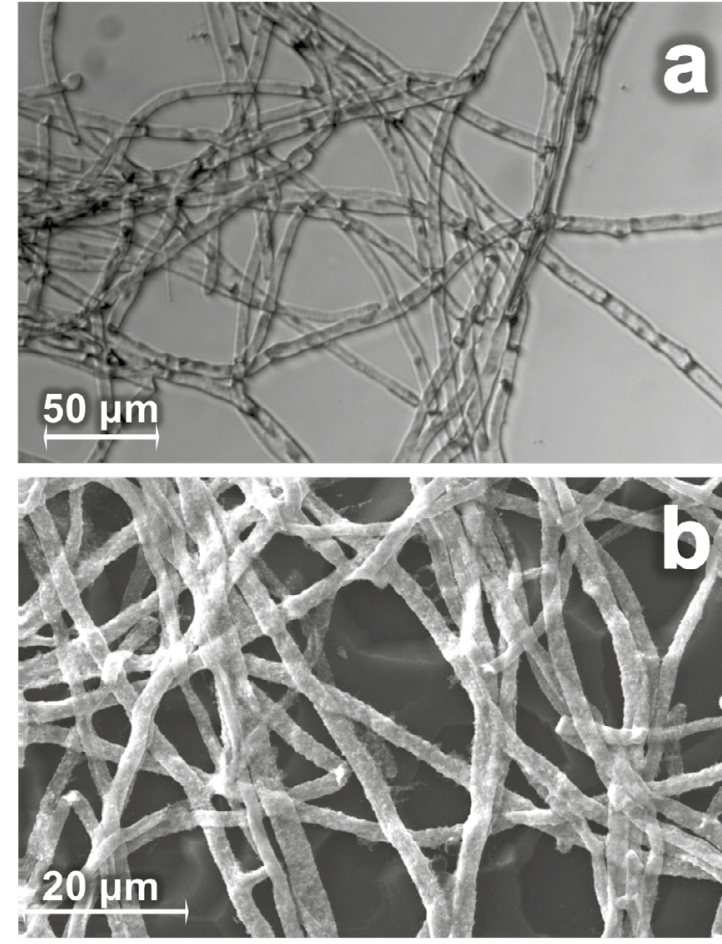

Figure 1. Optical micrographs of Aspergillus aculeatus before addition of AgNP and AuNP (a); scanning electron micrographs of the fungus after the addition of colloidal dispersions (b).

biotemplate. It is possible that subsequent multilayers were formed due to the entropic contribution while the Brownian motion was responsible for the nanoparticles migration from the medium to the hyphae surface. ${ }^{22}$ However, these successive layers cannot be simply explained in terms of chemical bonds between nanoparticles and the fungus. Therefore, some attractive interaction should occur between these nanoparticles mediated by molecules secreted by the fungus $^{23-25}$. Gold or silver nanoparticles keep their colloidal stability indefinitely, but small changes in ionic strength, temperature or dielectric constant can destabilize the colloidal suspension owing to their influence on the diffuse electric double layer around each nanoparticle. Usually, filamentous fungi respond to chemical composition secreting several molecules, including hydrophobin proteins that modifies the surface tension of fungus hyphae. ${ }^{25}$ In our studies, we observed that the formation of subsequent multilayers could be associated to the influence of the secondary metabolites produced by living fungus due the stress caused by the presence of metal nanoparticles, since we did not observe nanoparticles deposited on the surface of dead fungus. ${ }^{23,24}$ The interaction of these hydrophobic molecules with colloidal nanoparticles seems to be responsible by the deposition of successive layers of nanoparticles on the fungus surface.

After one week, we removed the AgNP dispersion from the medium with alive fungus and added the second dispersion of AuNP aiming to form a hybrid material consisting of an 
internal layer of silver covered by an external layer of gold. Figure 2 shows the SEM image of this material and their respective EDX element mapping. The red, blue and green colors identify the AgNP and AuNP nanoparticles and the silicon substrate, respectively. It is worth noting that gold and silver nanoparticles were present and well dispersed throughout the hyphae.

Nevertheless, the microtubule composition taken from different regions of the same mycelial mass was not homogeneous. In fact, additionally to the microtubules composed of silver and gold nanoparticles shown in Fig.2, we identified regions with only AgNP (Fig.3) and others solely with AuNP (Fig.4). Although the culture medium was completely removed before adding new colloidal dispersions of nanoparticles, we consider the citrate used to reduce and stabilize the nanoparticles influenced the hyphal growth. Probably, the reason for the presence of three distinct regions with different compositions was the impossibility to stop the fungus growth, fed by the citrate from the colloidal dispersions of nanoparticles. ${ }^{13}$ The first region composed of AgNP was localized in the center of the mycelial mass. The second region, covered with multilayers of both gold and silver nanoparticles, was found in the intermediate segments of the hyphae. The third region with only AuNP occurred in the border of mycelial mass.
Although the undeniable potential use of these materials in several technological fields, the existence of such heterogeneities is, obviously, an obstacle for their wide application. For this reason, it is of fundamental importance to understand the relationship between the assembling mechanism and the final heterostructure obtained. Figure 5 illustrates a possible explanation of this irregular recovering. According to the morphology of the mycelium, the hyphae growth occurs uniquely in one direction from the center to outside. Cells present inside the mycelium do not contribute to the fungus growth, although they provide nutrients to the peripheral cells. Usually, fungi grows not in function of the number of cells, such as bacteria, but due the number of hyphae tips and the amount of nutrients provided by the medium. ${ }^{26}$ During the assembly process, nanoparticles firstly came into contact with the fungus when the AgNP dispersion was added onto the Erlenmeyer flask containing the mycelial mass. Naturally, these AgNP formed a uniform layer on the hyphae surface, even when the fungi was growing, due the excess of AgNP in the medium, which maintained the concentration of nanoparticles almost homogeneous around the fungus during the deposition, resulting in a uniform kinetic of deposition. Later, when the second dispersion of gold nanoparticles was introduced substituting the silver nanoparticles, a second layer of AuNP was formed on the
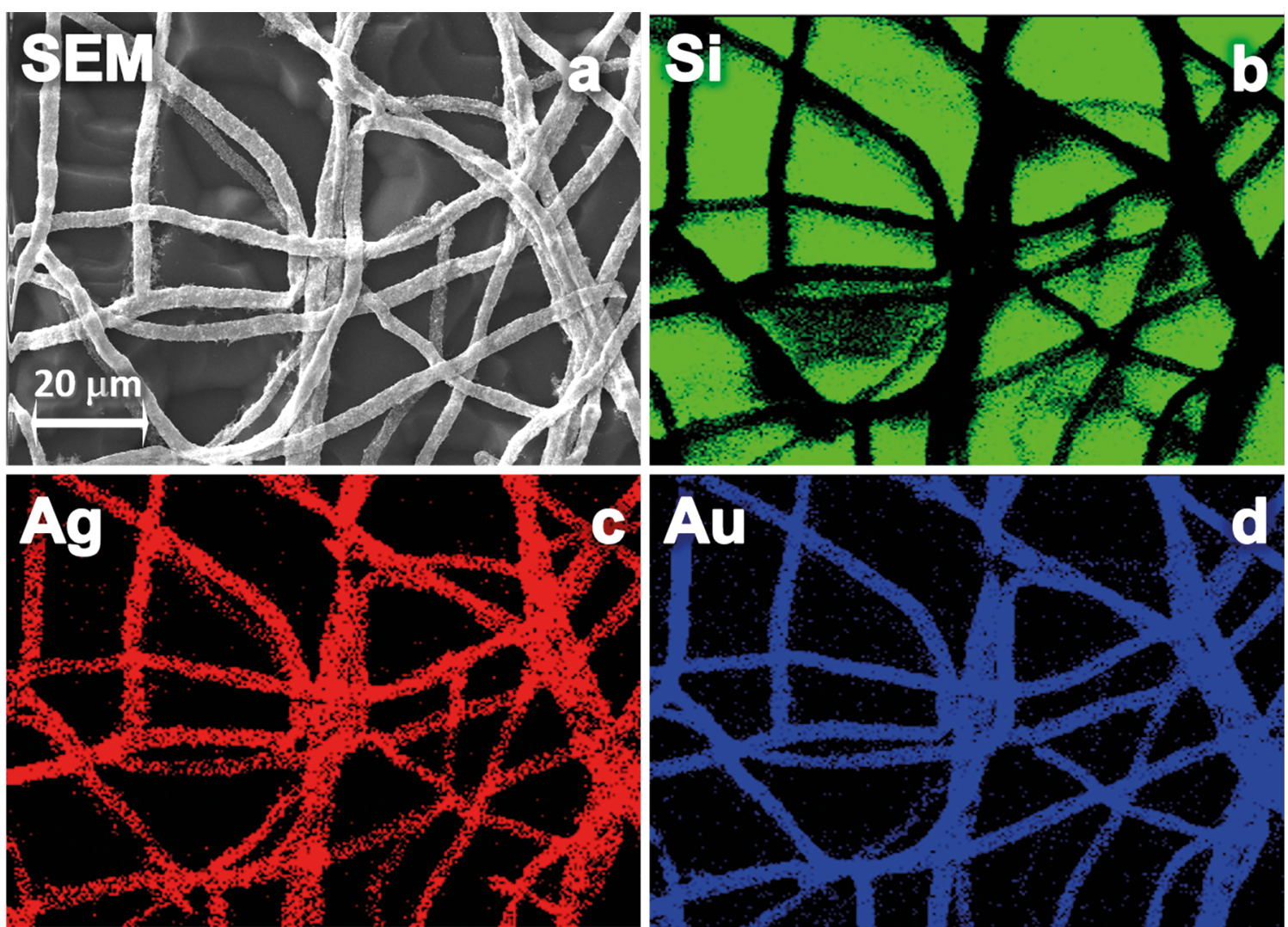

Figure 2. SEM images and EDX mapping in 2D of the elements (b) Si, (c) Ag and (d) Au that compose the intermediate segments of the hyphae filamentous fungus Aspergillus aculeatus covered with gold and silver nanoparticles. Hyphae was deposited on a silicon substrate. 

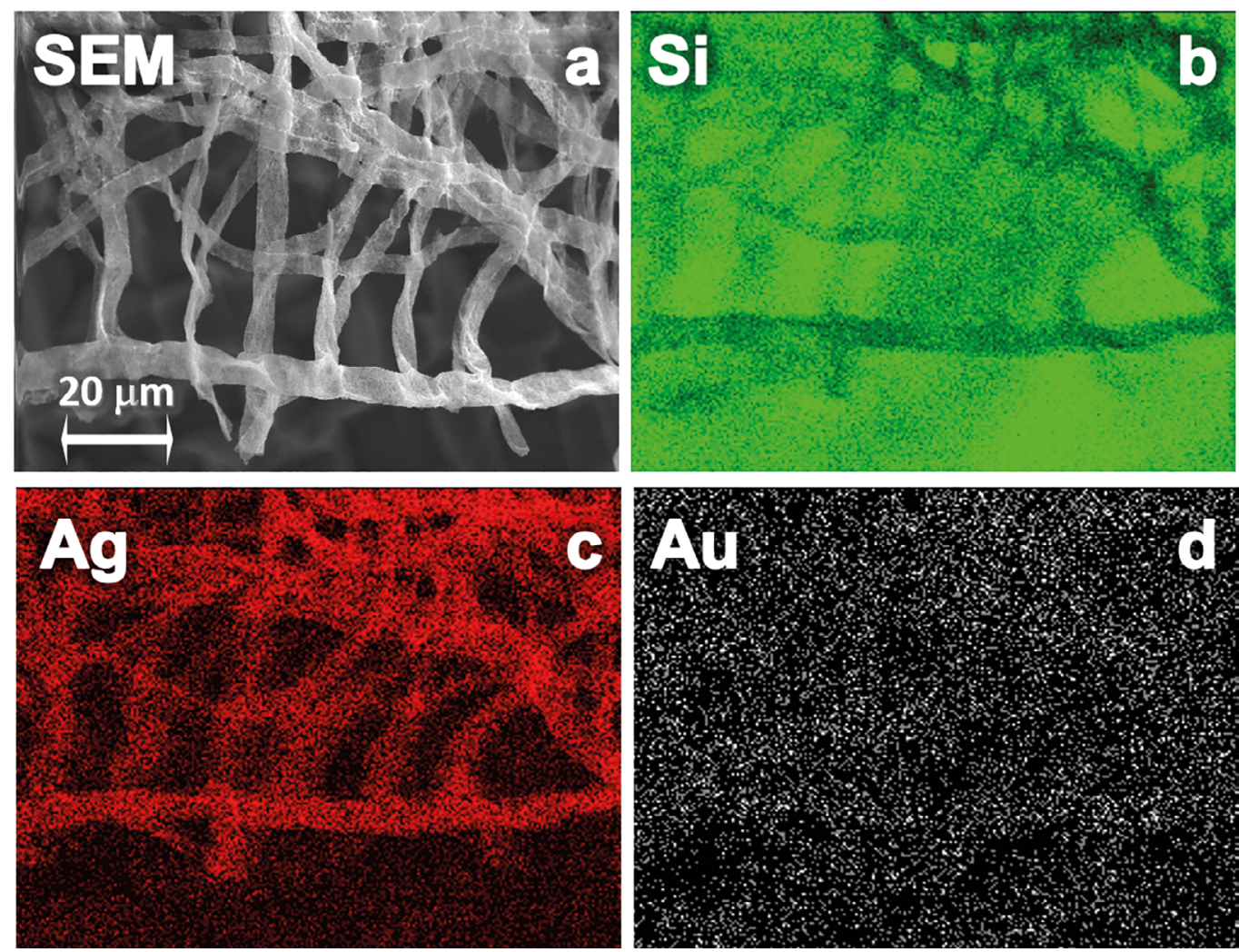

Figure 3. SEM images and EDX mapping in 2D of the elements (b) Si, (c) Ag and (d) Au that compose the filamentous fungus Aspergillus aculeatus localized in the center of the mycelial mass. Note that this region of fungus is covered solely with silver nanoparticles.
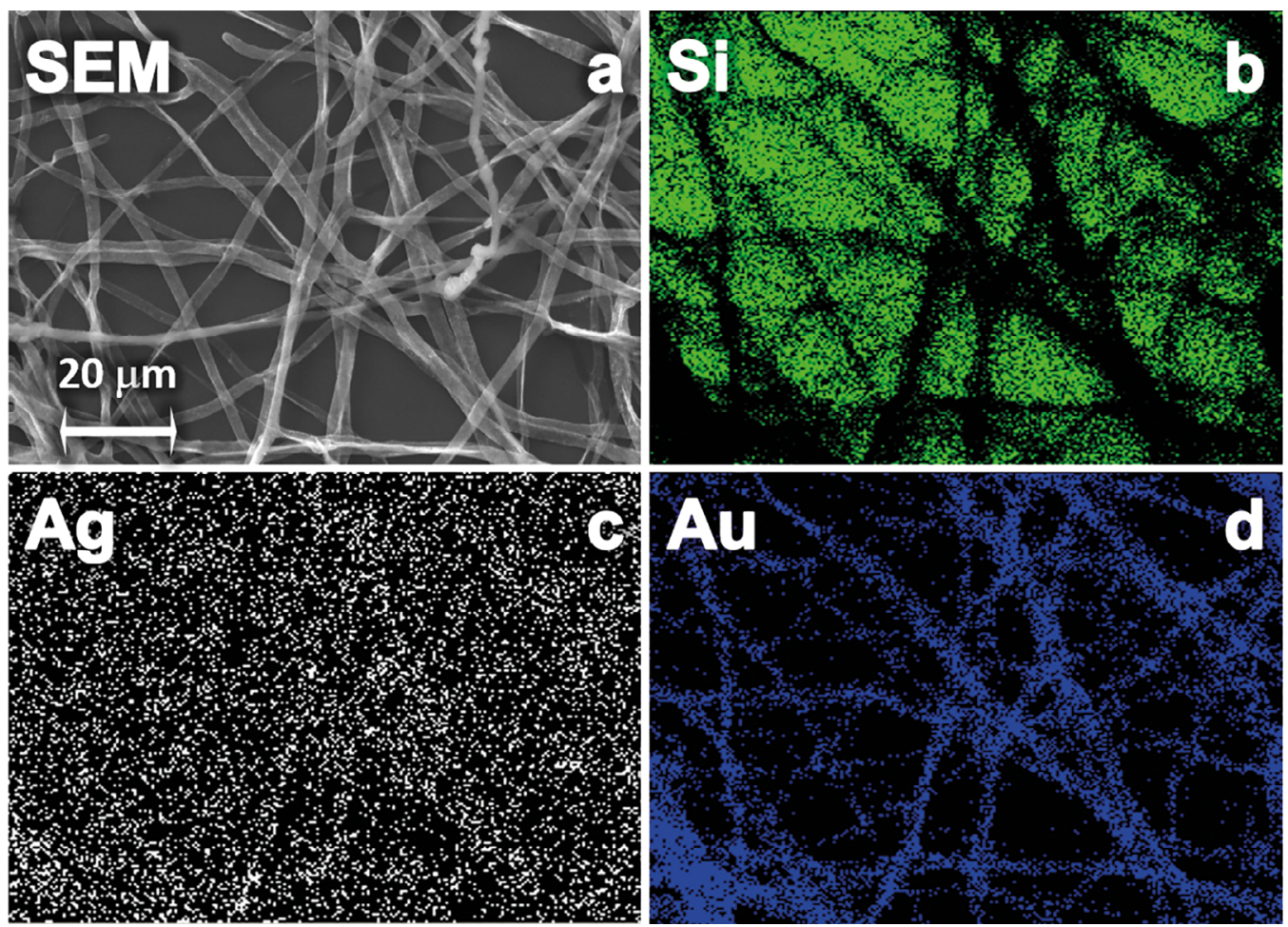

Figure 4. SEM images and EDX mapping in 2D of the elements (b) Si, (c) Ag and (d) Au that compose the filamentous fungus Aspergillus aculeatus in the border of mycelial mass covered exclusively with gold nanoparticles. 


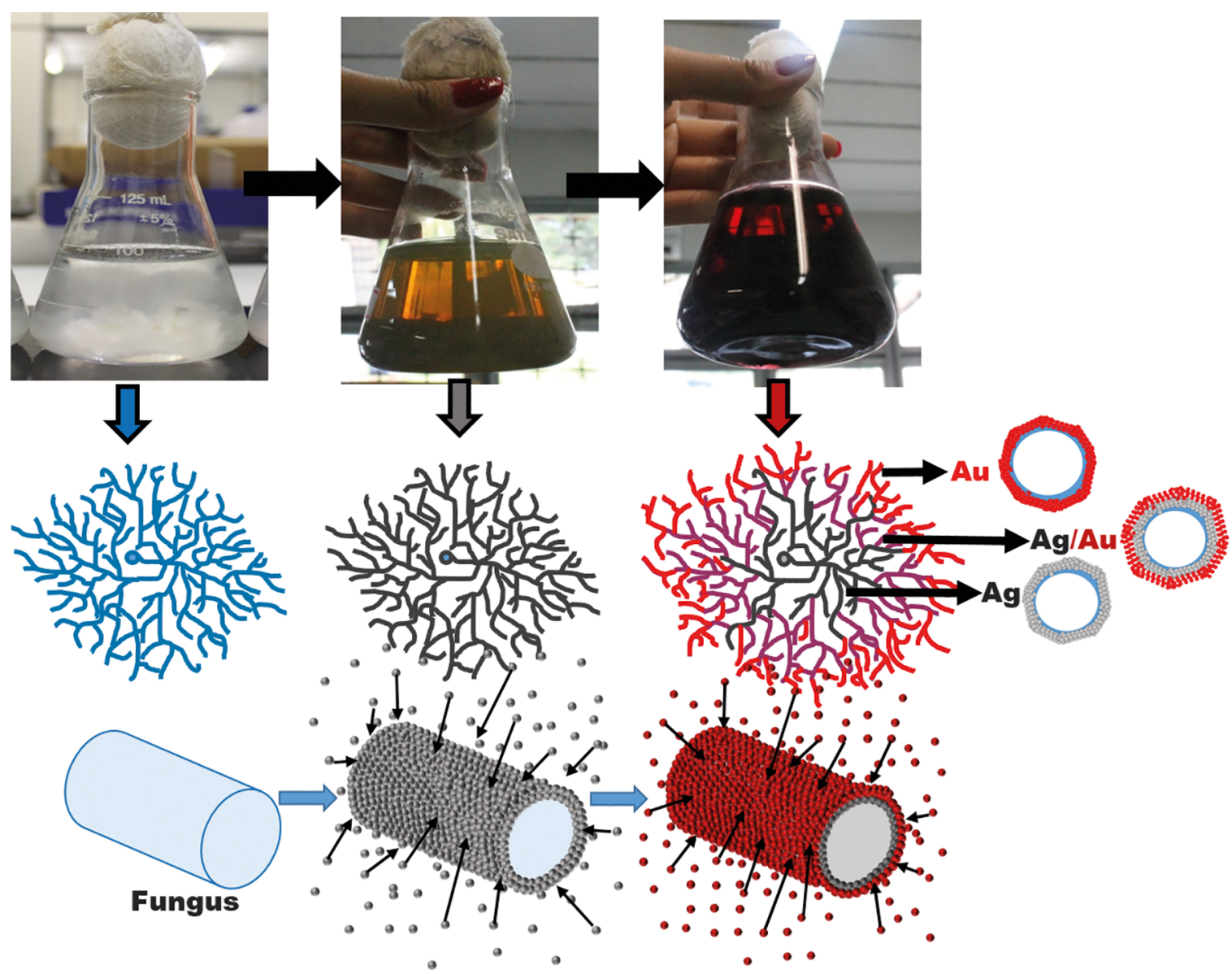

Figure 5. Ilustrative scheme of the different composition regions of the hyphae. Center of the hyphae is composed by AgNP. In an intermediated region, AgNP and AuNP. In the tips, AuNP only.

first layer of AgNP, as expected. However, in the presence of the nutrient citrate, hyphae were still growing, resulting in new surfaces without any AgNP. These new surfaces were covered exclusively by gold nanoparticles. Therefore, at least, two regions should be identified, one formed by multiple layers of AuNP and $\mathrm{AgNP}$ and a second of containing exclusively AuNP deposited on the new fungus filaments formed after removed silver nanoparticles. These two regions of pure gold microtubules at the border of mycelium and silver-gold microtubules in intermediary segments are in agreement with our observation. Moreover, due the fungus growth, the mycelium center became inaccessible to new nanoparticles in consequence of the tortuous path through the matted filament web, mainly considering that most of nanoparticles were captured by the hyphae surface during the diffusion process. This barrier effect preserved the mycelium center and, consequently, the microtubules initially formed inside it consisting exclusively of AgNP were inaccessible to new nanoparticles of gold coming from outside, which explain the third region of silver microtubules.

A key issue in nanotechnology is the fabrication of identical materials in large scale. Reproducibility and uniformity are fundamental aspects of any technology related to nanomaterials. ${ }^{23,24,27}$ Although the synthesis of colloidal nanocrystals appears to be simple, supra-nanoparticles assemblies is still a challenge. ${ }^{28}$ For this reason, the use of biological templates to control the assembly of nanoparticles allows the fabrication of functional materials through the exploration of cooperative and synergetic effects. Fungi morphologies are determined by their genetic code and by the environment characteristics. In fact, filamentous fungi exhibit variable responses to fungal strain and growth media, which include the amount and the composition of nutrients, $\mathrm{pH}$, temperature and humidity. ${ }^{23-25}$ In fixed condition, fungi will reproduce the morphological and physico-chemical behaviour, which will guarantee the production of materials of identical performance. On the other hand, growing fungi biotemplates in different volumes can introduce problems in scale-up that will interfere with the material quality. Perhaps, the best strategy to produce large amount of these hybrid materials with superior reproducibly and homogeneity could be increasing the number of modular reactors than by the use of larger reaction vessels, mainly considering that the formation of these microtubules is slow and exhibits low 
thermal hazard potential. This strategy is largely flexible to adjust the production to demand and client specification, which is particularly advantageous for fine chemical or biotechnological industries, where the production amounts are often small. ${ }^{29}$

\section{Conclusion}

Nanostructured functional materials were constructed in a multilayer process using two different noble metal nanoparticles, which evidently can be expanded to systems constituted of different nanoparticles or more than three layers. This approach opens new technological applications where metal heterojunctions play important role, such as catalysis and optical devices. However, the influence of the hyphae growth led to the formation of distinct regions in the same mycelium, which in turn generated some compositional heterogeneity. Evidently, the key point was related to the feeding ability of fungi to grow in presence of citrate. Perhaps, nanoparticles can be generated using other reducing agent than citrate since the simple fungus neutralization is not an option due the impossibility to form microtubules on dead fungus. On the other hand, the extraordinary capacity of these hybrid material to form an efficient barrier to the nanoparticle diffusion can be explored in fields related to environment remediation, depollution and active selective membranes in advanced materials for human and veterinary health.

\section{Acknowledgements}

The financial support of FAPESP (Grant 2015/13958-3 and 2013/07296-2), CNPq, CAPES is gratefully acknowledged. We also acknowledge Dr. Aurea M. L. de Moraes for the assistance in identifying the fungus.

\section{References}

1. Zhou JC, Gao Y, Martinez-Molares AA, Jing C, Yan D, Lau J, et al. Microtubule-based gold nanowires and nanowires array. Small. 2008;4(9):1507-1515.

2. Gopinathan P, Ashok AM, Selvakumar R. Bacterial flagella as biotemplate for the synthesis of silver nanoparticle impregnated bionanomaterial. Applied Surface Science. 2013;276:717-722.

3. Rosi NL, Thaxton CS, Mirkin CA. Control of Nanoparticle Assembly by Using DNA-Modified Diatom Templates. Angewandte Chemie International Edition. 2004;43(41):55005503 .

4. Liu G, Shao Y, Ma K, Cui Q, Wu F, Xu S. Synthesis of DNA-templated fluorescent gold nanoclusters. Gold Bulletin. 2012;45(2):69-74

5. Sugunan A, Melin P, Schnürer J, Hilborn JG, Dutta J. NutritionDriven Assembly of Colloidal Nanoparticles: Growing Fungi Assemble Gold Nanoparticles as Microwires. Advanced Materials. 2007;19(1):77-81.
6. Li Z, Chung SW, Nam JM, Ginger DS, Mirkin CA. Living Templates for the Hierarchical Assembly of Gold Nanoparticles. Angewandte Chemie International Edition . 2003;42(20):23062309 .

7. Gorup LF, Longo E, Leite ER, Camargo ER. Moderating effect of ammonia on particle growth and stability of quasi-monodisperse silver nanoparticles synthesized by the Turkevich method. Journal of Colloid and Interface Science. 2011;360(2):355-358.

8. Ziegler C, Eychmüller A. Seeded Growth Synthesis of Uniform Gold Nanoparticles with Diameters of 15-300 nm. Journal of Physical Chemistry C. 2011;115(11):4502-4506.

9. Frens G. Controled Nucleation for the Regulation of Particle Size in Monodisperse Gold Suspensions. Nature. 1973;241:2022.

10. Bigall NC, Eychmüller A. Synthesis of noble metal nanoparticles and their non-ordered superstructures. Philosophical Transactions of the Royal Society A. 2010;368(1915):1385-1404.

11. Niemeyer CM. Nanoparticles, Proteins, and Nucleic Acids: Biotechnology Meets Materials Science. Angewandte Chemie International Edition. 2001;40(22):4128-4158.

12. Sharma S, Srivastava S. Gold microwires based amperometric biosensor exploiting microbial architecture. Biosensors \& Bioelectronics. 2013;50:174-179.

13. Bigall NC, Reitzig M, Naumann W, Simon P, van Pée KH, Eychmüller A. Fungal Templates for Noble-Metal Nanoparticles and Their Application in Catalysis. Angewandte Chemie International Edition. 2008;47(41):7876-7879.

14. Monteiro DR, Silva S, Negri M, Gorup LF, Camargo ER, Oliveira R, et al. Silver colloidal nanoparticles: effect of matrix composition and structure of Candida albicans and Candida glabrata. Journal of Applied Microbiology. 2013;114(4):11751183.

15. Monteiro DR, Gorup LF, Takamiya AS, Ruvollo-Filho AC, Camargo ER, Barbosa DB. The growing importance of materials that prevent microbial adhesion: antimicrobial effect of medical devices containing silver. International Journal of Antimicrobial Agents. 2009;34(2):103-110.

16. Sabah A, Kumar P, Mohammed WS, Dutta J. Visible-LightInduced Direct Gold Microwires by Self-Organization of Nanoparticles on Aspergillus niger. Particle \& Particle Systems Characterization. 2013;30(5):473-480.

17. Fontes AM, Geris R, Santos AV, Pereira MG, Ramalho JGS, Silva $\mathrm{AF}$, et al. Bio-inspired gold microtubes based on the morphology of filamentous fungi. Biomaterials Science. 2014;2:956-960.

18. Forzza RCEA, Baumgratz JFA, Bicudo CEM, Carvalho Jr AA, Costa A, Costa DP, et al., orgs. Catálogo de Plantas e Fungos do Brasil. Rio de Janeiro: Instituto de Pesquisas Jardim Botânico do Rio de Janeiro; 2010.

19. Zhang S. Fabrication of novel biomaterials through molecular self-assembly. Nature Biotechnology. 2003;21(10):1171-1178.

20. Castro-Longoria E, Vilchis-Nestor AR, Avalos-Borja A. Biosynthesis of silver, gold and bimetallic nanoparticles using the filamentous fungus Neurospora crassa. Colloids and Surfaces B: Biointerfaces. 2011;83(1):42-48. 
21. Wu N, Heidelberg A, Boland JJ. Mechanical properties of ultrahighstrength gold nanowires. Nature Materials. 2005;4(7):525-529.

22. Sabah A, Dakua I, Kumar P, Mohammed WS, Dutta J. Growth of templated gold microwires by self organization of colloids on Aspergillus niger. Digest Journal of Nanomaterials and Biostructures. 2012;7(2):583-591.

23. Kubo AM, Gorup LF, Amaral LS, Rodrigues-Filho E, Camargo ER. Kinetic Control of Microtubule Morphology Obtained by Assembling Gold Nanoparticles on Living Fungal Biotemplates. Bioconjugate Chemistry. 2016;27(10):2337-2345.

24. Kubo AM, Gorup LF, Toffano L, Amaral LS, Rodrigues-Filho E, Mohan H, et al. Nanostructured Assemblies of Gold and Silver Nanoparticles for Plasmon Enhanced Spectroscopy Using Living Biotemplates. Colloids and Interfaces. 2017;1(1):4.
25. Sopandi T, Wardah A, Surtiningsih T, Swandi A, Smith JJ. Utilization and optimization of a waste stream cellulose culture medium for pigment production by Penicillium spp. Journal of Applied Microbiology. 2012;114(3):733-745

26. Alexopoulos CJ, Mims CW, Blackwell MM. Introductory Mycology. New York: Wiley; 1996. 880 p.

27. Juárez J, Cambón A, Topete A, Taboada P, Mosquera V. OneDimensional Magnetic Nanowires Obtained by Protein Fibril Biotemplating. Chemistry. 2011;17(26):7366-7373.

28. Tian C, Cordeiro MAL, Lhermite J, Xin HL, Shani L, Liu M, et al. Supra-Nanoparticle Funcional Assemblies through Programmable Stacking. ACS Nano. 2017;11(7):7036-7048.

29. Jensen KF. Microreaction engineering - is small better? Chemical Engineering Science. 2001;56(2):293-303. 